\title{
Uremic Encephalopathy: MR Imaging Findings and Clinical Correlation
}

\author{
(1)D.M. Kim, ㄴ.1.H. Lee, and (1)C.J. Song
}

\begin{abstract}
BACKGROUND AND PURPOSE: Uremic encephalopathy is a metabolic disorder in patients with renal failure. The purpose of this study was to describe the MR imaging findings of uremic encephalopathy.
\end{abstract}

MATERIALS AND METHODS: This study retrospectively reviewed MR imaging findings in 10 patients with clinically proved uremic encephalopathy between May 2005 and December 2014. Parameters evaluated were lesion location and appearance; MR signal intensity of the lesions on TIWI, T2WI, and T2 fluid-attenuated inversion recovery images; the presence or absence of restricted diffusion on diffusion-weighted images and apparent diffusion coefficient maps; and the reversibility of documented signal-intensity abnormalities on follow-up MR imaging.

RESULTS: MR imaging abnormalities accompanying marked elevation of serum creatinine (range, $4.3-11.7 \mathrm{mg} / \mathrm{dL}$ ) were evident in the 10 patients. Nine patients had a history of chronic renal failure with expansile bilateral basal ganglia lesions, and 1 patient with acute renal failure had reversible largely cortical lesions. Two of 6 patients with available arterial blood gas results had metabolic acidosis. All basal ganglia lesions showed expansile high signal intensity (lentiform fork sign) on T2WI. Varied levels of restricted diffusion and a range of signal intensities on DWI were evident and were not correlated with serum Cr levels. All cortical lesions demonstrated high signal intensity on T2WI. Four patients with follow-up MR imaging after hemodialysis showed complete resolution of all lesions.

CONCLUSIONS: The lentiform fork sign is reliable in the early diagnosis of uremic encephalopathy, regardless of the presence of metabolic acidosis. Cytotoxic edema and/or vasogenic edema on DWI/ADC maps may be associated with uremic encephalopathy.

ABBREVIATIONS: ARF = acute renal failure; $\mathrm{DM}=$ diabetes mellitus; $\mathrm{LFS}=$ lentiform fork sign; $\mathrm{SI}=$ signal intensity; $\mathrm{UE}=$ uremic encephalopathy

U remic encephalopathy is a metabolic disorder that occurs in patients with acute or chronic renal failure. This toxicmetabolic encephalopathy is a complication resulting from endogenous uremic toxins in patients with severe renal failure. The pathogenesis is complex and unclear.

Knowledge concerning a uremic encephalopathy (UE)-specific imaging feature, the so-called lentiform fork sign (LFS), is limited to case reports. The LFS may also be present in metabolic acidosis from any cause, including end-stage renal disease, methanol intoxication, and the dialysis disequilibrium syndrome. ${ }^{1-6}$ This study was undertaken to describe the MR imaging features in

Received August 3, 2015; accepted after revision February 12, 2016.

From the Department of Radiology, Chungnam National University Hospital, Chungnam National University School of Medicine, Daejeon, Korea.

Please address correspondence to In Ho Lee, MD, Department of Radiology, Chungnam National University Hospital, Chungnam National University School of Medicine, 282 Munhwa-ro, Jung-gu, Daejeon 301-721, Korea; e-mail: leeinho1974@hanmail.net

三 Indicates article with supplemental on-line table.

http://dx.doi.org/10.3174/ajnr.A4776 the brains of 10 patients with UE, with the aim of identifying common imaging features and potentially clarifying the possible pathophysiology of UE.

\section{MATERIALS AND METHODS}

This retrospective study was reviewed and approved by our institutional review board. The study included 10 patients $(9$ men and 1 woman; mean age, 58 years; age range, 17-76 years) with clinically proved UE who had abnormalities on brain imaging when examined between May 2005 and December 2014. Eleven potential patients were identified by a search of the imaging archive at our institution for patients who had the LFS on MR imaging. Imaging findings and medical records of these 11 patients were reviewed. Two of the patients were excluded because of other toxic/metabolic diseases, such as extrapontine myelinolysis. One additional patient who underwent MR imaging after a clinical diagnosis of UE was subsequently identified and included. UE diagnoses in patients were clinical and supported by laboratory findings showing deterioration of renal function and typical neurologic complications. Other possible causative 
conditions, such as cerebral stroke, drug-induced movement disorder, neurodegenerative disease, or other metabolic disorders ${ }^{7,8}$ were excluded by chart review.

The medical records of the 10 patients were reviewed for the history and duration of diabetes mellitus (DM); hypertension; the cause of renal failure and categorization into acute renal failure (ARF) or chronic renal failure; presenting symptoms; laboratory findings (serum blood urea, nitrogen, and creatinine levels; metabolic acidosis on arterial blood gas); treatment; and recurrence. Metabolic acidosis was diagnosed on arterial blood gas if the $\mathrm{pH}$ was low $(<7.35)$ and the bicarbonate levels were decreased $(<24$ $\mathrm{mmol} / \mathrm{L}$; normal range, $19-24 \mathrm{mmol} / \mathrm{L}$ ). All patients underwent MR imaging within a few hours of the onset of symptoms. The MR imaging was performed by using a $3 \mathrm{~T}$ scanner (Intera Achieva; Philips Healthcare, Best, the Netherlands) and a $1.5 \mathrm{~T}$ scanner (Signa Excite; GE Healthcare, Milwaukee, Wisconsin). The protocol on the 3T scanner included T2-weighted imaging (TR/TE, 3000/80 ms; section thickness, $5 \mathrm{~mm}$; section gap, 1.5 $\mathrm{mm}$; FOV, $220 \times 220 \mathrm{~mm}$; matrix, $400 \times 300$; number of signals acquired, 1), FLAIR (TR/TE, 11,000/125 ms; section thickness, 5 $\mathrm{mm}$; section gap, $1.5 \mathrm{~mm}$; FOV, $220 \times 220 \mathrm{~mm}$; matrix, $316 \times$ 242; number of signals acquired, 1), T1-weighted imaging (TR/ TE, 2000/10 ms; section thickness, $5 \mathrm{~mm}$; section gap, $1.5 \mathrm{~mm}$; FOV, $220 \times 220 \mathrm{~mm}$; matrix, $260 \times 166$; number of signals acquired, 1), and axial 2D gradient recalled-echo imaging (TR/TE, 453/14 ms; flip angle, $20^{\circ}$; section thickness, $5 \mathrm{~mm}$; section gap, $1.5 \mathrm{~mm}$; FOV, $220 \times 220 \mathrm{~mm}$; matrix, $275 \times 275$ ). The protocol on the $1.5 \mathrm{~T}$ scanner included T2-weighted imaging (TR/TE, 3600/120 ms; section thickness, $5 \mathrm{~mm}$; section gap, $1.5 \mathrm{~mm}$; FOV, $220 \times 220 \mathrm{~mm}$; matrix, $256 \times 256$; number of signals acquired, 2), and T1-weighted imaging (TR/TE, 450/10 ms; section thickness, $5 \mathrm{~mm}$; section gap, $1.5 \mathrm{~mm}$; FOV, $220 \times 220 \mathrm{~mm}$; matrix, $320 \times 190$; number of signals acquired, 2).

DWI with b-values of 0 and 1000 was performed in the axial plane, by using 3 orthogonal directions of diffusion-sensitizing gradients, combined into isotropic images. Other parameters included TR/TE, 4300/46 ms; section thickness, 3 mm; section gap, $1 \mathrm{~mm}$; FOV , $240 \times 240 \mathrm{~mm}$; matrix, $128 \times 126$; number of signals acquired, 1.

All MR imaging data were retrospectively evaluated by 2 experienced radiologists for lesion location; appearance; MR signal intensity on T1WI, T2WI, and FLAIR images; DWI and ADC maps; the presence or absence of hemorrhage; and the reversibility of signal intensity (SI) on follow-up MR imaging. The LFS was defined as bilateral, expansile, and symmetric hyperintensity in the basal ganglia surrounded by a more hyperintense rim delineating the lentiform nucleus on T2WI.

\section{RESULTS}

Clinical and imaging findings of UE in 10 patients are presented in the On-line Table. Nine of 10 patients had a history of underlying chronic renal failure, ranging from 3 months to 14 years. The remaining patient had ARF. Seven of 9 patients had underlying diabetic chronic renal disease (long-term DM; range, 5-30 years), and 2 of 9 patients had underlying hypertensive chronic renal disease without DM. All 9 patients with chronic renal failure regularly received hemodialysis. Seven patients had hypertension (systolic pressure range, $140-178 \mathrm{~mm} \mathrm{Hg}$ ), and 2 patients were normotensive on emergency department visits. The blood pressure of 1 patient was not evaluated in the outpatient clinic. All patients had acute neurologic symptoms as follows: movement disorder $(n=7)$, dysarthria $(n=4)$, and altered mental status $(n=1)$. All patients had uremia; blood chemistry analyses showed marked elevation of blood urea nitrogen (range, 31.7-69 mg/dL; healthy average after dialysis, $8-20 \mathrm{mg} / \mathrm{dL}$ ) and $\mathrm{Cr}$ (range, $4.3-$ $11.7 \mathrm{mg} / \mathrm{dL}$; healthy average after dialysis, $0.8-1.2 \mathrm{mg} / \mathrm{dL}$ ). Other serum electrolyte and glucose levels were not significantly abnormal, excluding other causes of encephalopathy. Of 5 patients with available arterial blood gas and bilateral basal ganglia lesions, 1 (patient 2) had a mild metabolic acidosis ( $\mathrm{pH}, 7.31$; bicarbonate, 21.7), and 4 patients had a normal $\mathrm{pH}$ level. One patient (patient 7) with $\mathrm{ARF}$ and cortical lesions on MR imaging also had mild metabolic acidosis ( $\mathrm{pH}, 7.3$; bicarbonate, 19.0).

In 9 of 10 patients with chronic renal failure, MR imaging showed bilateral, expansile, symmetric basal ganglia lesions with increased SI on T2WI, FLAIR, and/or ADC maps, compatible with vasogenic edema (LFS, Fig $1 A$ ). Four of 9 patients showed diffuse mildly increased SI on DWI without restricted diffusion in the bilateral basal ganglia lesions, and 2 showed focal restricted diffusion, corresponding to cytotoxic edema, in the globus pallidi (Fig $2 A,-B$ ). The remaining 5 patients showed normal SI on DWI in the bilateral basal ganglia. One patient had restricted diffusion in the bilateral globus pallidus and left putamen (Fig $3 A,-B$ ). There was no significant difference among any of the patients regarding clinical findings, including serum Cr level (5.63-11.7 $\mathrm{mg} / \mathrm{dL}$ in the group of 4 patients versus $4.3-10.8 \mathrm{mg} / \mathrm{dL}$ in the group of 5 patients).

Decreased SI on T1WI was noted in the lesions of 5 patients. There were no hemorrhages in the deep gray matter on T2WI or gradient recalled-echo images and no significant white matter abnormality in any patient.

Two of the 10 patients (patients 4 and 7) showed cortical involvement on T2WI and FLAIR. Two patients had markedly high systolic blood pressure (systolic blood pressure, $178 \mathrm{~mm} \mathrm{Hg}$; diastolic blood pressure, 86 and $58 \mathrm{~mm} \mathrm{Hg}$, respectively) on emergency department visits. One patient (patient 4) who had hypertensive chronic renal disease showed multifocal relatively symmetric, gyriform high SI on T2WI/FLAIR images and low SI on T1WI in the bilateral frontal and parieto-occipital lobes (Fig 1C) and the bilateral basal ganglia (LFS; Fig $1 A$ ) without restricted diffusion on ADC maps. Another patient (patient 7) who had ARF demonstrated multifocal gyriform high T2 SI lesions in the bilateral frontal, parieto-occipital, and right temporal lobes and subtle nodular high T2 SI in the right putamen without restricted diffusion on ADC maps. All patients showed improvement of various neurologic symptoms, normalization of serum Cr levels, and decreased serum blood urea nitrogen levels by $>60 \%$ after intensive hemodialysis. In 4 patients, follow-up MR imaging, ranging from 40 days to 3 years later, showed complete resolution of imaging abnormalities (Fig $4 A,-B$ ).

\section{DISCUSSION}

$\mathrm{UE}$ is a neurologic complication associated with ARF or chronic renal failure. It is likely caused by the effects of neurotoxic com- 

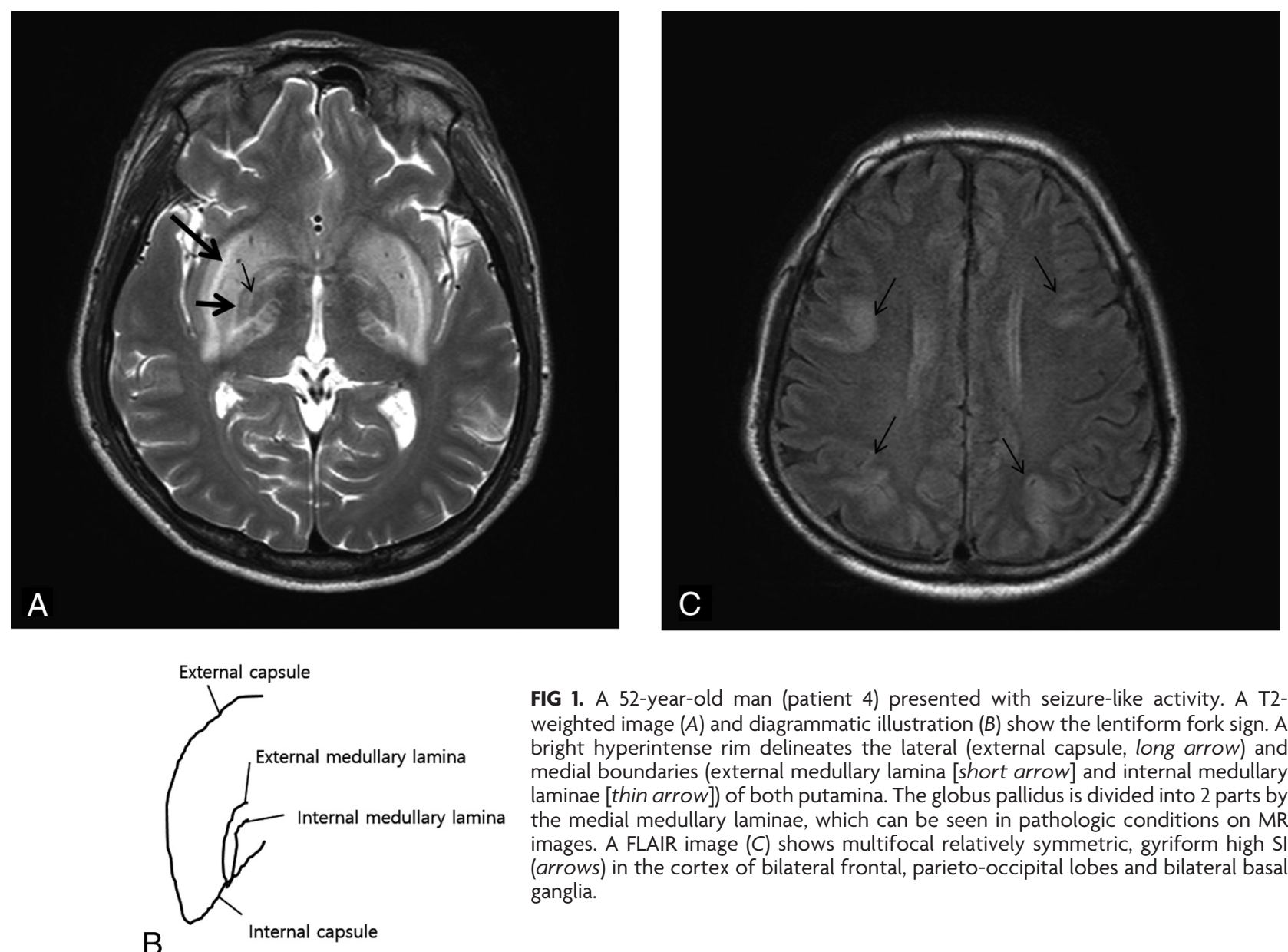

FIG 1. A 52-year-old man (patient 4) presented with seizure-like activity. A T2weighted image $(A)$ and diagrammatic illustration $(B)$ show the lentiform fork sign. A bright hyperintense rim delineates the lateral (external capsule, long arrow) and medial boundaries (external medullary lamina [short arrow] and internal medullary laminae [thin arrow]) of both putamina. The globus pallidus is divided into 2 parts by the medial medullary laminae, which can be seen in pathologic conditions on MR images. A FLAIR image (C) shows multifocal relatively symmetric, gyriform high SI (arrows) in the cortex of bilateral frontal, parieto-occipital lobes and bilateral basal ganglia.

B

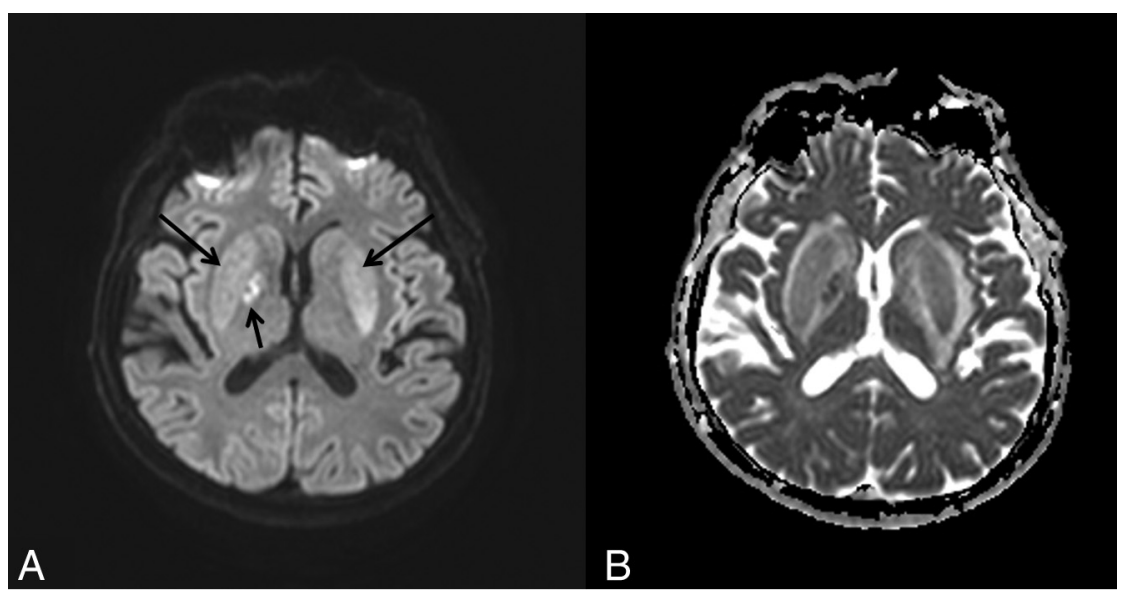

FIG 2. A 67-year-old man (patient 5) presented with unsteadiness. DWI (A) and an ADC map (B) show diffuse mildly increased SI (long arrows) in the whole bilateral basal ganglia lesion on DWI with focal restricted diffusion (short arrow), corresponding to cytotoxic edema in the right globus pallidus.

pounds. Accumulation of uremic toxins such as guanidine compounds (eg, creatinine and guanidine) might stimulate the neurotoxic effect of excitatory $N$-methyl-D-aspartate receptors, and concomitant inhibition of inhibitory $\gamma$-aminobutyric acid receptors may play an important role in the etiology of UE. ${ }^{9}$ It may be an interruption of polysynaptic pathways and alterations in excitatory-inhibitory amino acid balance. The manifestations of this brain disorder include various neurologic symptoms such as movement disorders (tremor, asterixis, myoclonus), cognitive disorders, and alteration in mental status. ${ }^{10,11}$

UE presents 3 patterns of imaging findings: basal ganglia involvement, ${ }^{12-14}$ cortical or subcortical involvement, ${ }^{15,16}$ and white matter involvement. ${ }^{17,18}$ Basal ganglia involvement is more common in Asian patients with DM. The LFS in patients with UE often affects Asians (from Taiwan, ${ }^{12,13}$ Korea, ${ }^{13,14,18}$ Japan $^{19}$ ). DM may make the basal ganglia more vulnerable to uremic toxins because of endothelial dysfunction in cerebral vessels (vascular autoregulatory dysfunction $^{20}$ ), and uremic toxin inhibits mitochondrial function with destruction of the pallidum and putamen. ${ }^{21}$ Cortical involvement is a category of posterior reversible leukoencephalopathy syndrome, ${ }^{22}$ and DM does not have a great effect on the involvement. ${ }^{18}$ Reversible intracerebral disease entities mediated by vascular autoregulatory dysfunction can be classified into 2 distinct groups: hypertensive encephalopathy and UE. ${ }^{22}$ However, posterior reversible encephalopathy syndrome is also seen in the uremic conditions in which endothelial toxins are thought to play a role. ${ }^{16}$ Posterior reversible encephalopathy syndrome is a clinical-radiologic syndrome 


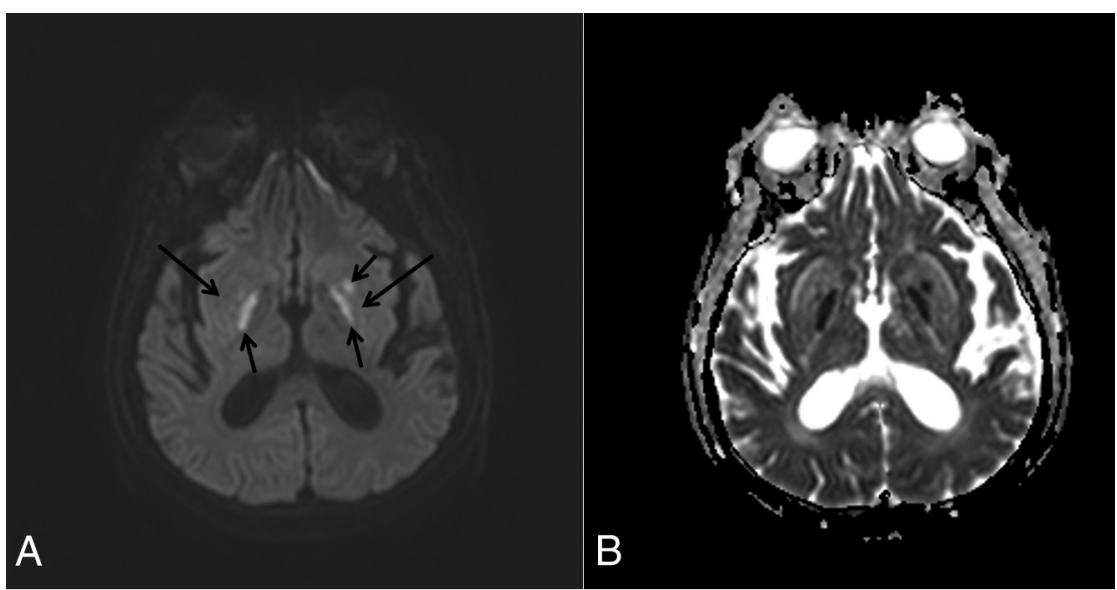

FIG 3. A 70-year-old man (patient 6) presented with involuntary movement. DWI (A) and an ADC map (B) show normal SI (long arrows) in the bilateral basal ganglia on DWI with restricted diffusion (short arrows) in the bilateral globus pallidus and left putamen, compatible with areas of cytotoxic edema in the lesions.
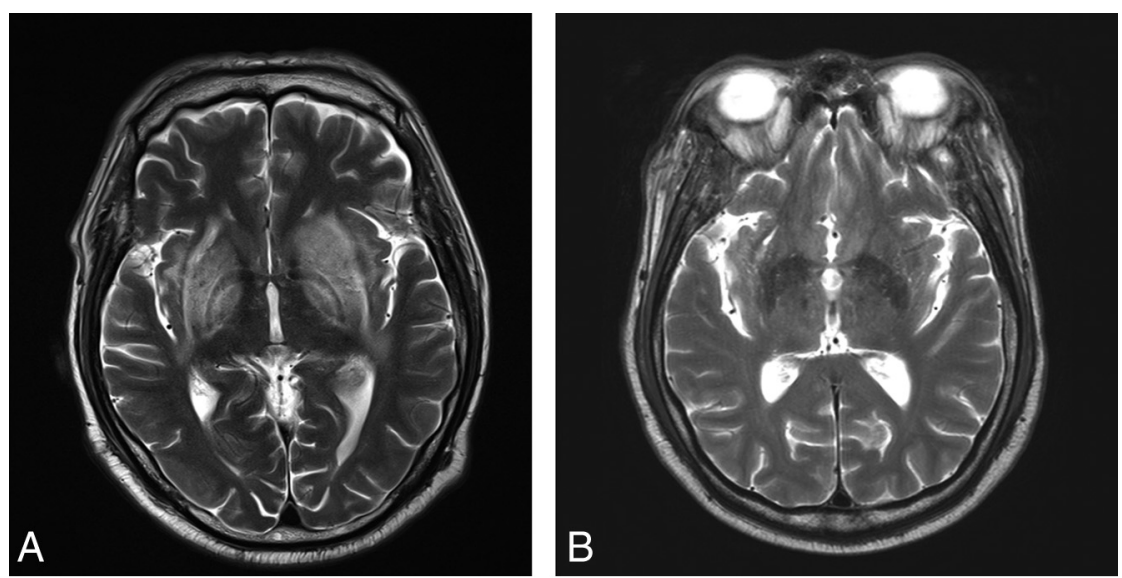

FIG 4. A 56-year-old man (patient 3) presented with dysarthria. Initial T2WI (A) shows the lentiform fork sign in the bilateral basal ganglia. Six months later, follow-up T2WI (B) shows complete resolution of the lesions.

characterized by headache, confusion, seizures, and visual disturbance and is thought to be secondary to hypertension. It classically occurs in the cortical or subcortical regions of the dominant parieto-occipital lobes, holohemispheric watershed zones, superior frontal sulcal area, and, rarely, the brain stem or basal ganglia. ${ }^{16,23,24} \mathrm{MR}$ imaging typically demonstrates vasogenic edema in the subcortical white matter and cortex. Cytotoxic edema has been described in a relatively lower percentage of patients with posterior reversible encephalopathy syndrome. ${ }^{25}$

Central-variant posterior reversible encephalopathy syndrome had been described as brain stem or basal ganglia involvement that spares the cerebral cortex and subcortical white matter. ${ }^{26}$ Imaging findings of central variant posterior reversible encephalopathy syndrome include symmetric or asymmetric vasogenic edema (the pons and basal ganglia [caudate nucleus, globus pallidus, and not resulting in the LFS]; and, rarely, the thalamus, posterior limb of the internal capsule, cerebellum, periventricular white matter) without any foci of reduced diffusivity and multiple microhemorrhages in the affected regions on susceptibility-weighted images. ${ }^{26}$ These imaging findings are substantially different from the imaging findings of UE. White matter involvement is the rarest form and occurs in the supratentorial white matter regions. This form might be considered a urea cycle disorder rather than UE.

The LFS has been described on T2WI/FLAIR images as a hyperintense rim delineating the lateral (external capsule) and medial (internal capsule, internal and external medullary laminae) boundaries of both putamina, resembling a fork (Fig 1B). ${ }^{1}$ A literature review $^{1}$ reported that the LFS was found in only 1 of 21 patients with UE admitted during a 10 -year period in a retrospective single-institution study, without note of the absence or presence of DM. However, data from the same literature review showed that 10 of 11 patients with UE who had the LFS had diabetes, and 1 patient with diabetic ketoacidosis had the LFS, implicating diabetes in the production of these characteristic lentiform lesions. In this study, 7 of 9 patients with UE who had the LFS during a 10-year period had long-term DM. The results may indicate a higher prevalence of the LFS in Asian patients with DM and UE.

Metabolic acidosis associated with disruption of the blood-brain barrier is an essential element in the generation of the LFS, ${ }^{1,2}$ and this sign may be attributable to differences in metabolic vulnerability between neurons of the basal ganglia and the astrocytes of surrounding white matter. Although UE is associated with metabolic acidosis, in this study, 1 of 5 patients with the LFS had mild metabolic acidosis, and the other 4 patients had normal $\mathrm{pH}$ levels on arterial blood gas. These findings are different from the results of a literature review that showed that metabolic acidosis is the basis of the LFS, and the absence of LFS might be reflective of a normal $\mathrm{pH} .{ }^{1}$ We propose that the LFS can be seen in UE regardless of the presence of metabolic acidosis, though coexistent metabolic acidosis may exacerbate the damage of neurons in the basal ganglia.

In this study, T2WI/FLAIR images showed uniform imaging findings. Diffuse vasogenic edema in the bilateral basal ganglia and/or cortical lesions represents increased interstitial fluid from a disruption of autoregulation. These results correspond well with those of the earlier studies. ${ }^{13,14}$ There was no significant difference between groups with diffuse mildly increased SI on DWI without restricted diffusion and others with normal SI on DWI in basal ganglia lesions.

Three of 9 patients who underwent DWI showed cytotoxic edema within the vasogenic edema of the basal ganglia, and all 3 lesions had cytotoxic edema in the unilateral or bilateral globus pallidus. Thus, the globus pallidus may be more vulnerable to cytotoxic damage than the putamen in UE. The globus pallidus is thought to be a vulnerable area of toxic/metabolic injury because of its high energy requirements and resultant sensitivity to mitochondrial and nuclear dysfunction. ${ }^{21,27}$ Toxic/metabolic enceph- 
alopathy, including carbon monoxide, cyanide, cocaine, opiate, and manganese intoxication, and Wilson disease involve selective vulnerability of pallidal neurons. ${ }^{28-31}$ However, the vulnerability of the globus pallidus in UE has not been established, though cytotoxic edema in the globus pallidus has been described in UE. ${ }^{13,14}$ Several pathologic studies reported cytotoxic edema in posterior reversible encephalopathy syndrome associated with fibrinoid necrosis and microinfarction $^{32}$ and suggested a causal relationship between cytotoxic edema and larger areas of vasogenic edema. ${ }^{33}$ However, in this study, there was no significant difference in the extent of vasogenic edema between groups with cytotoxic edema and other groups with vasogenic edema in basal ganglia lesions.

Cortical or subcortical lesions of UE are predominantly found in the parieto-occipital lobes. ${ }^{16}$ Cortical lesion in patients with UE may share pathophysiologic and imaging features with posterior reversible encephalopathy syndrome. Combined clinical features (the presence of neurologic impairment, renal dysfunction, renal failure, DM, metabolic acidosis, systemic hypertension, eclampsia, pre-eclampsia, vascular disease, autoimmune disease, or cancer chemotherapy) and imaging features (the presence of bilateral basal ganglia involvement with LFS, vasogenic/cytotoxic edema in affected regions, or microhemorrhage) should be considered in the differential diagnosis of UE and posterior reversible encephalopathy syndrome.

UE in renal failure is reversibly associated with hemodialysis or peritoneal dialysis, which removes neurotoxic compounds. ${ }^{34}$ In this study, all patients showed improvement of their neurologic symptoms, normalization of serum Cr levels, decreased serum blood urea nitrogen levels by more than $60 \%$, and complete resolution of imaging abnormalities on available follow-up MR imaging after intensified hemodialysis.

There are some limitations to this study. First, the sample size was small. Follow-up MR imaging was not performed in the patients who had restricted diffusion (cytotoxic edema) in their basal ganglia lesions. We cannot comment on the reversibility of these lesions. The sensitivity/specificity of the LFS for UE was not determined because of selection bias in this study. Finally, this study has not reliably demonstrated that the more severe the UE, the more likely it is to demonstrate the LFS on MR imaging, also due to selection bias.

\section{CONCLUSIONS}

The LFS on T2WI/FLAIR imaging is important in the early diagnosis of UE, regardless of the presence of metabolic acidosis. Cytotoxic edema and/or vasogenic edema on DWI and ADC mapping may be helpful findings associated with UE.

\section{REFERENCES}

1. Kumar G, Goyal MK. Lentiform fork sign: a unique MRI pictureis metabolic acidosis responsible? Clin Neurol Neurosurg 2010;112: 805-12 CrossRef Medline

2. Grasso D, Borreggine C, Perfetto, F, et al. Lentiform fork sign: a magnetic resonance finding in a case of acute metabolic acidosis. Neuroradiol J 2014;27:288-92 CrossRef Medline

3. Fabiani G, Teive HA, Munhoz RP. Lentiform fork sign and fluctuating, reversible parkinsonism in a patient with uremic encephalopathy. Mov Disord 2013;28:1053 CrossRef Medline

4. Blanco M, Casado R, Vazquez F, et al. CT and MR imaging findings in methanol intoxication. AJNR Am J Neuroradiol 2006;27:452-54 Medline

5. Arora V, Nijjar IB, Multani AS, et al. MRI findings in methanol intoxication: a report of two cases. Br J Radiol 2007;80:e243-46 CrossRef Medline

6. da Rocha AJ, Maia AC Jr, da Silva CJ, et al. Lentiform fork sign in a child with dialysis disequilibrium syndrome: a transient MRI pat- tern which emphasizes neurologic consequence of metabolic acidosis. Clin Neurol Neurosurg 2013;115:790-92 CrossRef Medline

7. Roos KL. Emergency Neurology. New York: Springer-Verlag; 2012: xii

8. Barton B, Zauber SE, Goetz CG. Movement disorders caused by medical disease. Semin Neurol 2009;29:97-110 CrossRef Medline

9. Vanholder R, De Smet R. Glorieux G; European Uremic Toxin Work Group. Review on uremic toxins: classification, concentration, and interindividual variability. Kidney Int 2003;63:1934-43 CrossRef Medline

10. Seifter JL, Samuels MA. Uremic encephalopathy and other brain disorders associated with renal failure. Semin Neurol 2011;31: 139-43 CrossRef Medline

11. Etgen T. Kidney disease as a determinant of cognitive decline and dementia. Alzheimers Res Ther 2015;7:29 CrossRef Medline

12. Wang HC, Cheng SJ. The syndrome of acute bilateral basal ganglia lesions in diabetic uremic patients. J Neurol 2003;250:948-55 CrossRef Medline

13. Lee EJ, Park JH, Ihn Y, et al. Acute bilateral basal ganglia lesions in diabetic uraemia: diffusion-weighted MRI. Neuroradiology 2007;49: 1009-13 CrossRef Medline

14. Yoon $\mathrm{CH}$, Seok JI, Lee DK, et al. Bilateral basal ganglia and unilateral cortical involvement in a diabetic uremic patient. Clin Neurol Neurosurg 2009;111:477-79 CrossRef Medline

15. Schmidt M, Sitter T, Lederer SR, et al. Reversible MRI changes in a patient with uremic encephalopathy. J Jephrol 2001;14: 424-27 11730279

16. Tatsumoto N, Fujisaki K, Nagae H, et al. Reversible posterior leukoencephalopathy syndrome in a patient with severe uremic encephalopathy. Clin Nephrol 2010;74:154-58 CrossRef Medline

17. Prüss H, Siebert E, Masuhr, F. Reversible cytotoxic brain edema and facial weakness in uremic encephalopathy. J Neurol 2009;256: 1372-73 CrossRef Medline

18. Kang E, Jeon SJ, Choi SS. Uremic encephalopathy with atypical magnetic resonance features on diffusion-weighted images. Korean J Radiol 2012;13:808-11 CrossRef Medline

19. Okada J, Yoshikawa K. Matsuo H, et al. Reversible MRI and CT findings in uremic encephalopathy. Neuroradiology 1991;33:524-26 CrossRef Medline

20. Kwok RP, Juorio AV. Concentration of striatal tyramine and dopamine metabolism in diabetic rats and effect of insulin administration. Neuroendocrinology 1986;43:590-96 Medline

21. Albin RL. Basal ganglia neurotoxins. Neurol Clin 2000;18:665-80 CrossRef Medline

22. Port JD, Beauchamp NJ Jr. Reversible intracerebral pathologic entities mediated by vascular autoregulatory dysfunction. Radiographics 1998;18:353-67 CrossRef Medline

23. Bartynski WS, Boardman JF. Distinct imaging patterns and lesion distribution in posterior reversible encephalopathy syndrome. AJNR Am J Neuroradiol 2007;28:1320-27 CrossRef Medline

24. Kastrup O, Schlamann M, Moenninghoff C, et al. Posterior reversible encephalopathy syndrome: the spectrum of MR imaging patterns. Clin Neuroradiol 2015;25:161-71 CrossRef Medline

25. Gao B, Yu BX, Li RS, et al. Cytotoxic edema in posterior reversible encephalopathy syndrome: correlation of MRI features with serum albumin levels. AJNR Am J Neuroradiol 2015;36:1884-89 CrossRef Medline

26. McKinney AM, Jagadeesan BD, Truwit CL. Central-variant posterior reversible encephalopathy syndrome: brainstem or basal ganglia involvement lacking cortical or subcortical cerebral edema. AJR Am J Roentgenol 2013;201:631-38 CrossRef Medline

27. Sharrief AZ, Raffel J, Zee DS. Vitamin B(12) deficiency with bilateral globus pallidus abnormalities. Arch Neurol 2012;69:769-772 CrossRef Medline

28. Spadoni F, Stefani A, Morello, M, et al. Selective vulnerability of pallidal neurons in the early phases of manganese intoxication. Exp Brain Res 2000;135:544-51 CrossRef Medline 
29. Sharma P, Eesa M, Scott JN. Toxic and acquired metabolic encephalopathies: MRI appearance. AJR Am J Roentgenol 2009;193: 879-86 CrossRef Medline

30. Valk J, van der Knaap MS. Toxic encephalopathy. AJNR Am J Neuroradiol 1992;13:747-60 Medline

31. Alquist CR, McGoey R, Bastian F, et al. Bilateral globus pallidus lesions. J La State Med Soc 2012;164:145-46 Medline

32. Jacquot C, Glastonbury CM, Tihan T. Is posterior reversible enceph- alopathy syndrome really reversible? Autopsy findings 4.5 years after radiographic resolution. Clin Neuropathol 2015;34:26-33 CrossRef Medline

33. Li Y, Gor D, Walicki D, et al. Spectrum and potential pathogenesis of reversible posterior leukoencephalopathy syndrome. J Stroke Cerebrovasc Dis 2012;21:873-82 CrossRef Medline

34. Burn DJ, Bates D. Neurology and the kidney. J Neurol Neurosurg Psychiatry 1998;65:810-21 CrossRef Medline 